\title{
Determination of Recovery Effort for a Probabilistic Recovery System under Various Inventory Control Policies
}

\author{
Z. Pelin Bayındır ${ }^{1}$, Rommert Dekker, Eric Porras \\ Econometric Institute \\ Erasmus University Rotterdam \\ March, 2004
}

Econometric Institute Report 2004-08

\begin{abstract}
In this study we investigate the desired level of recovery under various inventory control policies when the success of recovery is probabilistic. Recovery process is modelled as a single stage operation and recovery effort is represented by the expected time spent for it. The effect of increasing recovery effort on the success probability together with unit cost of the operation is included by assuming general forms of dependencies. The desired level of recovery is investigated under four inventory control policies for a wide range of system parameters. In this article, we present our computational results and their managerial implications.
\end{abstract}

Keywords: Probabilistic recovery, inventory control

\footnotetext{
${ }^{1}$ Corresponding author. Tel:+31 1040886 32; fax:+31 104089162 E-mail address: bayindir@few.eur.nl (Z.P. Bayındır)
} 


\section{Introduction}

Recovery of used products has become an important issue for manufacturers in the past years, as a consequence of the increased awareness on the scarcity of resources and diminishing raw materials. In many countries there are laws and law proposals obliging manufacturers to take back their products after their useful life. Designing recovery systems that bring the used items into an as good as new condition has been taking attention of many researches with diverse backgrounds. In this study our main aim is to investigate the desired level of recovery effort in the economical sense.

In general in addition to environmental benefits, product recovery is seen as a cost reducing strategy. On the other hand, having a recovery practice requires new planning applications; mainly coordinating the traditional purchasing/production means with recovery operations due to the fact that these two sources are used to satisfy the same demand stream. In recent years a vast amount of academic knowledge has been accumulated in coordinating these decisions. de Brito [1, p.43] provides an up-to-date and extensive review of the literature.

One of the issues not investigated in detail is the fact that the recovery process is not perfect in the sense that not all of the items can be successfully recovered. To the best of our knowledge the imperfect recovery is only investigated by Ferrer [2]. He examines different situations about the information on the failure of recovery under a determinstic demand, single period setting. He includes a number of serial recovery operations and identifies the value of having the yield information in early stages of recovery. In addition the importance of the responsive suppliers is shown by a numerical experimentation.

Our study is motivated by an industrial practice in a European refinery (Rijneveld[5]). An important class kept in the inventory consists of (i.) expensive, (ii.) slow moving, (iii.) having high supplier lead time items. The system is also subject to returns which cannot be used as good as new ones. The returns go through a recovery process which consists of discrete testing and fixing operations, starting with roughest to the detailed ones. Our experience reveals that since the perception for recovery is positive in the economical sense, the objective of the recovery shop is to maximize the number of recovered items so that they have the tendency for practicing the recovery into finest detail. On the other hand, since the company's usual purchasing decisions do not take these returns into account in a formal way, the recovered 
items cause inventory accumulation for the serviceables that are used to satisfy demand. As most of these items are slow moving, their effects can be substantial in holding costs. Besides, the objective of maximizing throughput of recovery may not be an appropriate one considering the effect of increasing recovery effort on the success probability of recovery and costs.

The main objective of our study is to investigate the desired level of recovery for such a system. Our approach is in between tactical to operational in the sense that we consider the joint determination of recovery effort (that can be considered as a system design issue) and purchasing decisions (that is traditionally an operational decision). For the sake of generality and simplicity, we model recovery as a single stage operation with some unit cost and lead time. We consider expected time spent in recovery operation, i.e., expected lead time, as a measure of recovery effort and focus on the case where the success probability of recovery increases as the expected lead time increases. Increasing the expected lead time of recovery operation can be considered as performing further disassembly, testing and fixing operations, in other words increasing the number of steps that get to be more detailed. We also include the effect of increasing recovery effort on unit costs of recovery.

We specifically concentrate on the case where (i.) all of the items completing their usage time -which is stochastic with a known probability distribution- return to the system, and (ii.) the information on number of items currently in use is always available. Such a situation can occur under different practical environments. For instance consider any equipment, say an equipment used in the maintenance and repair of a certain machinery, that is used by the employees of the firm and prone to damage during the use. Whenever a need for use arises, a technician issues an equipment and after the use (s)he returns it. In general, the usage time is stochastic and the quality level of the used equipment is not as good as new. Similarly, you can think of any commercial product where all of the customers return the products at the end of the useful life time as obliged by the legislation and sales agreement. In these cases it is important to keep track of the number of items currently in use, in order to monitor the inventory system correctly. We assume that all of the issues and return information are perfectly recorded, therefore the number of items currently in use is known.

For the system described above we propose four distinct inventory control policies, and the desired level of recovery is investigated under different system conditions: expected life time of 
the product, expected supplier lead time, unit purchasing cost, sensitivity of success probability and unit cost to the increase in expected lead time of the recovery operation.

The outline of the paper is as follows: In Section 2, the technical assumptions are given together with model formulation. In Section 3, the results of the numerical study are presented and finally in Section 4 managerial insights gained by the study and the effect of employed assumptions are discussed.

\section{Technical Assumptions and Model Formulation}

In this section we discuss the specifications of the considered environment in detail. Proposed inventory control policies are discussed in Section 2.1, and a generic steady-state cost function is derived in Section 2.2. The notation summarized in Table 1 is used throughout the article.

\section{Place Table 1 here}

We consider a single item inventory system facing stochastic demand. We assume that the demand process is Poisson with rate $\lambda$ in accordance with representing a slow moving item. Unsatisfied demand is completely backordered. Each item demanded is returned after a stochastic usage time. This means that either the customers are the employees of the firm, or there is a sales agreement or a legislation dictating 100\% take-back. The information on the number of items currently in use is always available. Regardless of its age, each returned item goes into a recovery operation after which it is brought to an as-good-as new condition with a certain probability. Recovered items are used as the new ones supplied from an outside vendor. Supplier lead time is assumed to be stochastic with a known distribution. We further assume that the supplier lead time is independent of the number of outstanding orders. In other words, we ignore the possible capacity restrictions of the supplier; the lead time of each order is independent of its size and number of items currently on order.

Our main objective in modelling such a recovery system is to understand the desired level of recovery under different system conditions on cost, lead time and demand parameters. In real life recovery operations consists of several discrete test and fixing phases. As the number of phases involved increases, the items are investigated in more detail and consequently the probability of successful recovery increases. Our main aim is to understand to what extent 
the recovery operation should go in more detail considering the trade off between recovery probability and incurred higher cost and lead times.

For the sake of simplicity we model recovery operation as a single stage and consider expected time spent for it as a measure of recovery effort. It is assumed that the recovery process can be designed in terms of determining its expected lead time. As the expected lead time of recovery, $T_{1}$, increases, the probability of success at recovery increases with a decreasing rate. Therefore, in a sense we consider $T_{1}$ as a tactical level decision variable representing the recovery effort which corresponds to an increased number of recovery phases. The following form of dependency is assumed:

$$
p\left(T_{1}\right)=1-e^{-k_{p} T_{1}},
$$

where $p\left(T_{1}\right)$ is the probability that a returned item is successfully recovered for given $T_{1}$ and $k_{p}$ is a positive constant and can be interpreted as a measure of efficiency of the recovery operation. Notice that, all the items going through the recovery operation fails, i.e., $p\left(T_{1}\right)$ is zero, if the recovery operation is not performed, i.e., $T_{1}$ is zero. On the other hand, in order to successfully recover all of the returned items, i.e., in order for $p\left(T_{1}\right)$ to be 1 , expected lead time of the operation should be set to infinity. We assume that $p\left(T_{1}\right)$ is time stationary and does not depend on the age of the returned item.

We also include the effect of increasing recovery effort on unit costs. As in most of the real life applications, increasing the recovery effort increases unit cost of the operation. The following form is assumed:

$$
c_{r}\left(T_{1}\right)=c_{b} T_{1}^{k_{c}}
$$

where $c_{r}\left(T_{1}\right)$ is the unit cost of recovery for given $T_{1}$, and $k_{c}$ and $c_{b}$ are positive constants. Using this generic form, we are able to mimic different cost structures. Note that the constant $c_{b}$ can be interpreted as a base value of unit cost; that is the unit cost to be incurred when one unit of time is spent for each item on the average. On the other hand, the constant $k_{c}$ can be interpreted as the cost efficiency of the recovery operation. Regardless of the value of $k_{c}$ and $c_{b}$, when $T_{1}$ is set to zero, the unit cost of recovery is zero. 
For the $k_{c}$ values that are greater than 1 , the unit cost of recovery is convex in $T_{1}>0$, where the unit cost increases with an increasing rate. This type of cost structure can occur when the first steps of recovery do not require much additional effort in terms of energy and resource usage, and as the steps go in more detail they require more. For instance, the first steps may share the same precedence operations, and the more detailed steps require unique set-ups. This case can be considered like diseconomies of scale. On the other hand, in order to mimic the concave cost structure where the marginal cost of each additional time unit spent for recovery decreases as the time increases, $k_{c}$ should be set to a level that is less than 1 . This case can be encountered when the economies of scale exist: the marginal energy and resource usage requirement of each step diminishes as the number of steps increases.

In real life applications it may be hard to estimate the value of the parameters $k_{p}, k_{c}$ and $c_{b}$ from scratch. For the items that the manufacturer has some accumulated experience on the recovery, statistical analysis can be utilized to fit such a function. In addition, our general approach and solution procedure can be easily extended to different forms of cost and yield functions.

In order to visualize the effect of these parameters, Figure 1 and 2 are provided under $c_{b}=0.1$ and $c_{b}=0.5$, respectively. In these figures, we include 4 scenarios on the efficiency and sensitivity of unit costs to the expected time spent for recovery operation. The parameter settings for these four cases are given in Table 2. As it can be seen in Table 2, Case 1 and Case 2 correspond a situation where recovery operation is less efficient than Case 3 and Case 4 with respect to yield. On the other hand, under Case 2 and Case 4, unit recovery cost is more sensitive to recovery effort than under Case 1 and Case 3.

Place Table 2, Figure 1 and Figure 2 here

As it can be observed in Figure 1 and 2, in order to reach a recovery probability which is close to 1 , an expected lead time $\left(T_{1}\right)$ of around 9 is required when the system is inefficient, i.e., under Case 1 and 2. When the system is efficient with respect to recovery success, i.e., under Case 3 and 4 , a $T_{1}$ of around 2 is sufficient to reach an almost perfect recovery operation. It is obvious that the difference between the unit costs under convex and concave cost functions changes at $T_{1}=1$. While achieving an almost perfect recovery, the highest unit cost is incurred 
when the system is inefficient and has a convex cost structure, i.e., Case 2, whereas the lowest unit cost is incurred when the system is efficient and has a concave cost structure, i.e., Case 3. As it can be observed, $c_{b}$ has the highest effect on the magnitude of unit costs and the difference between unit costs under different cases depends on the parameter selection.

We assume that the throughput of the recovery process is independent of the workload of the facility, i.e., the servers performing recovery can be increased as the workload increases. If we consider the fact that in general recovery operations are labor sensitive and is not primary operation area of the manufacturers, it is reasonable to assume that the number of labors performing the operation can be increased as the workload increases. Nevertheless, our basic model can easily be extended to the case where there are finite number of resources and the effect of this assumption on the numerical study is discussed in Section 4.

\subsection{Cost Items and Proposed Inventory Control Policies}

The following cost items are included in the analysis

- variable cost of recovery,

- variable cost of purchasing,

- holding cost of work-in-process (WIP) in recovery operation,

- holding and backorder costs for the serviceable items.

Note that although we do not include an explicit disposal cost for the items that are not successfully recovered, it may be included in both recovery and purchasing costs.

We ignore all of the fixed costs associated with recovery and purchasing operations. Inclusion of fixed costs provide additional tradeoffs between recovery and purchasing by batching decisions. We ignore batching decisions in order to focus on the desired level of recovery under different basic system parameters.

Consistent with ignoring the fixed costs, we propose four different order-up-to $S$, type policies. Note that one may seek the optimal inventory control policy together with desired level of recovery utilizing Markov Decision Process approach. In order to apply such an approach, additional assumptions on the underlying distribution of the lead times (including the life-time 
of the product) are required. More important than that, such an approach requires to deal with more than two dimensional state space. Since our main aim is to understand the general tendencies and draw managerial insights, we prefer to deal with the problem under the proposed policies.

While proposing inventory control policies, we try to create an advantage for the recovery option so that our computational findings create some sort of upper-bound on the desired level of recovery. Notice that if the recovery process was perfect all of the items under the recovery operation would be in serviceable inventory after they received the required service. So, like the number of items on-order from the supplier they should be included in the inventory position information. Including these items in inventory position information creates an advantage for the recovery option in the sense that the inventory decisions assume that they would be successfully recovered. Similarly, since the items currently in use eventually return to the system, they may be considered as a part of inventory position information. This is based on the idea that if the life-time of the product is small with respect other lead times, in other words if there is a quick return situation, this piece of information is beneficial to use. Therefore we come up with two definitions for the inventory position information as given below:

$$
\begin{aligned}
& I P_{1}=O H-B+N_{0}+N_{1}+N_{2} \\
& I P_{2}=O H-B+N_{1}+N_{2}
\end{aligned}
$$

Notice that in both of the inventory position definitions net inventory of serviceables $(\mathrm{OH}-$ $B)$, number of items on order from the supplier $\left(N_{2}\right)$ and the WIP at recovery operation $\left(N_{1}\right)$ are included. On the other hand, the number of items currently in use is only included in $I P_{1}$.

Similar to inventory position information, we consider the following two different decision epoches for purchasing decisions.

- $E_{1}$ : failure at recovery operation,

- $E_{2}$ : demand occurrence.

Deciding purchasing upon each failure at recovery $\left(E_{1}\right)$, makes the recovery operation as the primary source of supply. We expect that under quick return and quick recovery process 
and high recovery probability, this may outperform the traditional decision epoch, i.e., demand occurrence, for purchasing decisions.

Considering all combinations of two inventory position information and two decision epoches for purchasing decisions, we come up with four inventory control policies given in Table 3. Therefore under each policy proposed at each corresponding decision epoch, corresponding inventory information is checked. If it is below the corresponding order-up-to level $S$, an order of size equal to shortfall of the corresponding inventory position from $S$ is given to the supplier.

\section{Place Table 3 here}

Note that one may come with more detailed inventory policies like considering both epochs as a decision epoch, and defining distinct order-up-to levels based on different inventory positions. However, in this study we restrict ourselves to this simple policies. Giving the conditions under which these policies performs better than others, one may utilize them as an initial policy for policy improvement or due to ease of implementation they can be utilized as a heuristic method.

\subsection{The Steady State Average Cost Function}

\subsubsection{Holding and Backorder Costs}

There are two stock points in the system; returns that are currently under recovery and serviceable inventory.

We assume an inventory value of the used items, $h_{1}$, when they are under recovery operation. The long run expected holding cost of WIP can be expressed as a product of unit holding cost rate and expected number of items under recovery as follows:

$$
T C_{W I P}\left(S, T_{1}\right)=T C_{W I P}\left(T_{1}\right)=h_{1} E\left[N_{1}\right] .
$$

Note that since we assume that all items completing their usage time go under recovery, inventory control parameters do not affect this cost item due to the fact that the arrivals to the recovery operation is driven only by the demand process.

At recovery, a unit cost of $c_{r}\left(T_{1}\right)$ is added to each recovered item. On the other hand, new items are purchased at a cost of $c_{p}$. Therefore, inventory value of recovered and new items are not necessarily the same. Determination of inventory value of returns and serviceables, that 
includes both recovered and brand new items, is subject to ongoing research. Teunter et. al. [6], Teunter and van der Laan [7] and van der Laan[8] show that setting holding cost rates is not straightforward under reverse logistics. These studies provide some specific results under the specific inventory control policies employed. Differentiating the serviceable inventory for recovered and brand-new items and assigning non-identical holding costs may be considered as an alternative solution. However, in that case one needs to deal with depot discipline issues. In this study we assume the traditional way of setting holding cost rates and average costing principle. Therefore holding cost of a recovered and a brand new item is $h_{1}+r c_{r}\left(T_{1}\right)$ and $r c_{p}$, respectively. Since, on the average $100 p\left(T_{1}\right) \%$ of the items are recovered, we give a weight of $p\left(T_{1}\right)$ to the holding cost of recovered items and $1-p\left(T_{1}\right)$ to the new ones. Therefore, unit holding cost of serviceables are:

$$
h\left(T_{1}\right)=\left[h_{1}+r c_{r}\left(T_{1}\right)\right] p\left(T_{1}\right)+r c_{p}\left(1-p\left(T_{1}\right)\right) .
$$

The long run probability distribution of net serviceable inventory is required to express the total holding and backorder costs associated with it.

$$
T C_{F G I}\left(S, T_{1}\right)=h\left(T_{1}\right) \sum_{i=0}^{\infty} i P(I=i)-b \sum_{i=-\infty}^{0} i P(I=i)
$$

where $P(I=i)$ is the long-run probability that net inventory for serviceables is equal to $i$ and certainly these probability terms depends on the inventory policy employed.

\subsubsection{Purchasing and Recovery Costs}

In the steady state, since all items demanded go under recovery operation, average recovery cost is independent of the inventory policy and related policy parameters. In the long run, unit cost of recovery is incurred with the demand rate. On the other hand, on the average approximately $100\left(1-p\left(T_{1}\right)\right) \%$ of demand is satisfied by new items. Therefore, average total variable cost of recovery and purchasing is independent of inventory policy and can be expressed as,

$$
T C_{V A R}\left(S, T_{1}\right)=T C_{V A R}\left(T_{1}\right)=\lambda\left[c_{r}\left(T_{1}\right)+\left(1-p\left(T_{1}\right)\right) c_{p}\right]
$$


The problem of finding the optimal level of recovery, $T_{1}$, and order-up-to level $S$ can be stated as:

$$
\begin{array}{ll}
\min & T C\left(S, T_{1}\right)=T C_{V A R}\left(S, T_{1}\right)+T C_{W I P}\left(S, T_{1}\right)+T C_{F G I}\left(S, T_{1}\right) \\
\text { s.t. } & T_{1} \geq 0 \\
& S=\{1,2, \ldots\}
\end{array}
$$

where the general form of functions $T C_{W I P}\left(S, T_{1}\right), T C_{F G I}\left(S, T_{1}\right)$, and $T C_{V A R}\left(S, T_{1}\right)$ are given in equations (1), (2) and (3).

Under only Policy I the long-run average cost associated with serviceables can be derived analytically. The details of this procedure, together with explicit expressions for functions $T C_{V A R}\left(S, T_{1}\right), T C_{W I P}\left(S, T_{1}\right)$ and $T C_{F G I}\left(S, T_{1}\right)$ are given in Appendix I.

For the other policies, in order to analytically derive the steady-state probabilities, as to seek the optimal policy, one needs to deal with a multi-dimensional infinite state space problem. Since our aim is to understand the general tendencies we rely on the simulation, and a simulation optimization tool, OptQuest for Arena, Version 5.0 (See [9] for product information). OptQuest is a generic optimizer based on Meta Heuristics. When it is used upon simulation package Arena, it searches input parameters of the simulation model defined as decision variables in a non-monotonous manner to find approximate optimal solution. We model the system under each three policies using Arena. Similar to analytical search procedure that is applied under Policy 1 (see Appendix I), instead of $T_{1} p\left(T_{1}\right)$ is considered to be a discrete decision variable with a small step size in range $[0,1)$, in addition to $S$.

\section{$3 \quad$ Numerical Study}

We carried out an extensive numerical study to investigate:

- the desired level of recovery under the four proposed inventory control policies,

- the effect of system parameters on the desired level of recovery,

- the relative performance of the proposed inventory control policies. 
A full factorial experiment is designed. The following parameters are kept unvaried: $r=0.2$, $b=20, c_{p}=1$. The levels of the varying factors are given in Table 4 .

\section{Place Table 4 here}

Notice that by using this experimental setting we cover (i.) four different cases on the efficiency structure given as an example in Section 2, i.e., Case 1 to Case 4, under high $\left(c_{b}=0.5\right)$ and low magnitude $\left(c_{b}=0.1\right)$ of unit recovery cost, (ii.) no $\left(h_{1}=0\right)$ and high inventory value of returns $\left(h_{1}=0.1\right)$, (iii.) fairly low $(\lambda=0.1)$ and very low $(\lambda=0.01)$ demand rate, (iv.) four different levels for the difference between expected supplier lead time and expected life-time of the product under all of which expected life-time is higher than the expected supplier lead time.

In the experiment we assume that the underlying distribution of all lead-times including life time of the item is Exponential. Each problem instance is solved under all four proposed inventory control policies. The optimal policy parameters, $\left(S, T_{1}\right)$, are found as explained in Appendix I under Policy I. On the other hand, under the other policies the simulation optimization procedure is utilized to find the approximate optimal policy parameters. All (approximate) optimal steady-state cost figures are within $5 \%$ relative precision.

In the following subsections the results obtained are discussed. The raw results are available upon request.

\subsection{Cost Comparison of Inventory Control Policies}

The performance of the proposed policies are investigated based on pairwise comparisons. For each problem instance considered, $95 \%$ confidence intervals are constructed for the difference between the expected average cost figures. (See Law and Kelton [4, p.587])

Based on these pairwise comparisons, the following observations are made

- When the expected life time of the item is very high with respect to supplier lead time, i.e., $T_{0}=100$, under all parameter setting considered, Policy IV outperforms the others.

- When the expected life time of the product is slightly higher than the supplier lead time, i.e., when $T_{0}=5$ and $T_{2}=0$, 
- when the item is very slow moving, i.e., $\lambda=0.01$, Policy III outperforms the others under all parameter settings considered.

- when the item is fairly slow moving, i.e., $\lambda=0.1$, Policy IV outperforms the others under all parameter settings considered except under Case 1 when $h_{1}=0$ and $c_{b}=0.1$ where Policy III outperforms Policy IV.

- When the expected life time of the product is closer to the supplier lead time, i.e., when $T_{0}=5$ and $T_{2}=3$,

- when the item is very slow moving, i.e., $\lambda=0.01$,

* when there is no holding cost for WIP at recovery process, i.e., when $h_{1}=0$, Policy I performs at least as good as others except when $c_{b}=0.5$ where Policy IV is the best.

* when there is a holding cost associated with WIP at recovery, i.e., $h_{1}=0.1$, Policy IV performs at least as good as others except when $c_{b}=0.5$ under Case 4 and when $c_{b}=0.1$ under Case 3 and 4 . In these exceptional cases Policy IV is outperformed by Policy I.

- when the item is fairly slow moving, i.e., $\lambda=0.1$, Policy IV outperforms the others under all parameter settings considered except the following cases:

* when $c_{b}=0.1$ and $h_{1}=0$ under Case 3 and 4 where there is no significant difference between the performance of the policies,

* when $c_{b}=0.1$ and $h_{1}=0.1$ under Case 3 and 4 where Policy III outperforms Policy IV.

These results are in the same direction as our initial assessment on the proposed inventory policies. When the expected life time of the product is high with respect to the expected supplier lead time (even if the difference is only 5 time units) considering recovery as a primary source of satisfying demand, consequently making the purchasing decisions upon the failure occurrence results in higher expected costs. Under such a condition, when the demand is very rare, i.e., when the item is very slow moving, the expected number of items currently in use is small, and time between two demand occurrence time is sufficiently large to have some recovered 
items. In these cases, considering items currently in use improves expected costs and Policy III outperforms Policy IV. On the other hand, when the demand rate is not so low, the number of items currently in use is larger, and having a recovered item between two demand occurrence is not high. In these cases, excluding the items currently in use improves the costs and Policy IV outperforms Policy III.

On the other hand, considering recovery as the primary source to satisfy demand, and making purchasing decisions upon failure of recovery improves expected cost figures, only when (i.) the lead time of the supplier is very close to the expected life time of the product, (ii.) the item is very slow moving, and (iii.) recovery option is cost attractive. Under these cases, Policy I outperforms the others. Our computational experience reveals that under such a setting the items currently in use should be included in inventory position information since there is no problem instance where Policy II outperforms I.

\subsection{Cost Improvement by Applying the Best Inventory Control Policy}

In this subsection, we investigate the difference in costs under proposed inventory control policies. We focus on the maximum difference that is the percentage cost improvement by the "best" policy over the "worst" one for each problem instance considered. The "best" and the "worst" policies are defined for each problem instance according to the following criteria:

The Best: The one which is as good as the others in all pairwise comparisons. If there is more than one policy satisfying the condition or if there is no significant difference between all of the pairs, we take the one giving the minimum expected cost regardless of the estimated standard deviation of the average cost.

The Worst: The one which is worse than all the others in all pairwise comparisons. If there is no such a policy satisfying the condition or if there is no significant difference between all of the pairs, we take the one giving the minimum expected cost regardless of the estimated standard deviation of the average cost.

We calculate the percentage improvement by the point estimate of the expected cost under the best policy over the worst one for each problem instance. Since we concentrate on the percent changes, we lose the confidence on these estimates in the statistical sense. Alternatively we 
might report the pairwise differences with the corresponding confidence intervals. However, in this case the results would depend on the magnitude of the specific problem parameters. Therefore, the percentage deviations are reported to represent general tendencies in the value of applying the best policy under different parameter settings.

In Table 5 and Table 6 , minimum, maximum and average percentage improvements are summarized for different problem clusters for $T_{0}=100$ and $T_{0}=5$, respectively. For each problem cluster, the corresponding case on and only one problem parameter given in column heading kept constant, and all levels of the other parameters are included.

\section{Place Table 5 and Table 6 here}

As it can be seen in Table 5 and Table 6 , the percentage cost difference is at least $1 \%$ in almost all of the problem settings considered. Applying the right inventory control policy significantly gets important as:

- the expected life time of the product, $T_{0}$,

- the expected supplier lead time, $T_{2}$

- the base unit cost of recovery, $c_{b}$,

- the holding cost of WIP at recovery operation, $h_{1}$,

- the demand rate, $\lambda$,

increases.

In general, applying the right inventory control policy is more important for the systems that are both inefficient and incurring a higher unit costs for the high recovery probabilities, i.e., Case 2. As it can be seen in Table 5 and Table 6 , although the minimum percent improvement may be very small for these systems, the average and maximum improvements are higher than others. The reason behind the small minimum improvements is due to the fact that most of the time under Case 2 the recovery is not utilized under most of the policies, and consequently expected costs are close to each other. On the other hand, the ones that are efficient in terms of both unit cost and yield, are the most robust ones with respect to applied inventory control policy. 


\subsection{Desired Recovery Probability under the Best Policy}

In order to asses the systems parameters under which the recovery option is attractive we consider the optimal $p$ value under the best policy. As in the previous subsection, we name the policy "best" if it is as good as the others in all pairwise comparisons. Whenever there is more than one such a policy for a specific problem instance considered, we consider the one under which the optimized $p$ value is higher.

In Table 7 and Table 8 minimum, maximum and average optimized recovery probabilities are summarized under different problem parameters for $T_{0}=100$ and $T_{0}=5$, respectively. These tables are prepared in the same manner as Table 5 and Table 6 .

\section{Place Table 7 and Table 8 here}

As it can be observed from these tables, in general desired recovery probability increases as

- the expected life time of the product, $T_{0}$, decreases

- the expected supplier lead time, $T_{2}$, increases

- the base unit cost of recovery, $c_{b}$, decreases,

- the holding cost of WIP at recovery operation, $h_{1}$, decreases

- the demand rate, $\lambda$, increases.

It can be observed that the desired level of recovery can be very high even if the recovery operation is inefficient with respect to yield, i.e., Case 1 and Case 2, when the base unit cost is low, and there is no holding cost associated with WIP at recovery operation. On the other hand, the average behavior shows that under Case 3 and Case 4, the desired level of recovery is the highest.

When we focus on the problem instances where the desired recovery probability is at least 0.90 under all four policies, we see that these are the instances where recovery option is cost attractive with respect to magnitude of the unit cost, i.e., $c_{b}=0.1$, and the system is efficient with respect to trade off between recovery effort and probability, i.e., $k_{p}=2$. When the inventory value of returns are negligible, i.e., $h_{1}=0$, and the cost increase is by factor of 0.5 , 
i.e., Case 3, under all parameter settings on demand, expected lead time and expected life time parameters, the desired level of recovery is at least 0.90 . On the other hand, when the cost and efficiency structure follows Case 4 , and $T_{0}=5$ (i.) when $T_{2}=3$ under both demand scenarios, and (ii.) when $T_{2}=0$ when demand is very slow moving, the desired level of recovery is at least 0.9 regardless of the selected inventory control policy. We observed that when the expected life time of the product is close to expected supplier lead time, i.e., $T_{0}=5, T_{2}=3$, when the cost and efficiency structure follows Case 3, and the item is fairly slow moving, desired recovery probability is at least 0.90 even if there is an inventory value of returns.

This analysis reveals that when the recovery operation is cost attractive with respect to the magnitude of the unit recovery cost and inventory value of returns, maximizing recovery success is in the same direction as minimizing total cost if the unit recovery costs do not increase dramatically with the recovery effort. The disadvantage of initial inventory value of returns can be compensated under efficient and less unit cost sensitive systems i.e, Case 3, when the expected supplier lead time is close to the expected life time of the product, for fairly low demand rate. Under such a condition, when the item is very slow moving, the desired level of recovery depends on the inventory control policy.

Among all, there is only one problem instance where all policies yield no recovery solution, i.e., optimized $p=0$. This is the case where $T_{0}=5, T_{2}=0, c_{b}=0.5, h_{1}=0.1, \lambda=0.1$ under Case 1. Probably, there are other cases where no recovery solution is the best one, but due to numerical errors involved they may not have been captured.

In order to examine the optimal recovery effort, that is the expected time spent for the recovery operation, we examine them in the same way as the desired recovery probability. In Table 9 and 10 minimum, maximum and average optimized $T_{1}$ values are summarized for $T_{0}=100$ and $T_{0}=5$, respectively. These tables are prepared in the same manner as Table 5 and Table 6 .

\section{Place Table 9 and Table 10 here}

The effect of the problem parameters on the optimized recovery effort is similar to the desired probability effort, since they are dependent variables. However, examining Table 9 and Table 10 is interesting to see the effort bared to spend. It is obvious that, when the system 
is efficient, i.e, Case 3 and 4 , with a very small effort, i.e, with with small amount spent on the average, high recovery probabilities are obtained. On the other hand, when the recovery option is cost attractive, and unit recovery cost is not very sensitive to recovery effort, even if the process is inefficient, i.e., Case 1, large lead times compared to the supplier lead time are spent to take this advantage.

Our computational experience reveals that, in addition to the cost attributes of the recovery operation, the relative difference between the expected supplier lead time and the expected life time has a great importance on both the performance of the policies and plausibility of recovery option. In order to further investigate this effect we concentrate on a second set of scenarios where the expected supplier lead time is greater than the expected life time of the product. The parameters, like in the primary set, $r=0.2, b=20, c_{p}=1$ are assumed. In this case we restrict ourselves to the case where the base cost for recovery is small, i.e., $c_{b}=0.1$, there is no inventory value of returns, i.e., $h_{1}=0$, and the item demand is very rare, i.e., $\lambda=0.01$ and expected life time is very small, $T_{0}=1$. On the efficiency and cost sensitivity of recovery operation, we consider two extreme cases, Case 1 and Case 4, and three levels for expected supplier lead time, $T_{2}=2,3$ and 4 , considered.

In this second set, it is observed that under Case 4, Policy I and under Case 1, Policy III performs at least as good as others based on all pairwise tests at a significance level 0.05. Since $T_{2}$ values considered are very close to each other, the optimized $T_{1}$ and $p$ values are also very close to each other under the best policy. Under all problems considered the desired recovery probability is around 0.85 , and in order to reach that level, an expected lead time of almost 3.9 and 1.1 are spent, under Case 1, and Case 4, respectively. These results reveal that when the recovery operation is cost attractive and the expected lead time of the supplier is large with respect to expected life time of the product, the items in use should be considered while making purchasing decisions, when the demand is very rare. On the other hand, if the recovery operation is not efficient, since the recovery effort would be high in terms of its lead time, the right decision epoch for purchasing is demand occurrence epoch. 


\section{Conclusion}

In this study we investigate the desired recovery effort when the recovery process is not perfect. The effect of increased recovery effort on both the unit costs and the success of recovery operation is included in an abstract model where the recovery operation is represented by a single stage. Increasing the expected lead time of this operation corresponds to an increase in the number of steps of recovery in real life applications. Four distinct inventory control policies that differ in the moment that the purchasing decision is made and in the way that inventory position upon which the purchasing decision is based is defined are proposed.

Our computational experience reveals the following managerial insights.

- Recovery is most attractive for the systems where

- the recovery operation is efficient in terms of gain obtained in success by increasing its lead time,

- the magnitude of unit recovery cost is small with respect to purchasing cost,

- unit recovery cost is not much sensitive to an increase in its lead time.

For these systems maximizing the recovery success is almost equivalent to minimize expected costs. Therefore, before trying to maximize the output of the recovery shop, the pros in terms of gains in recovery success and cons in terms of increased unit costs should be well assessed. In addition, the sensitivity of the desired level of recovery with respect to efficiency shows the importance of process improvement studies that involves various research areas from material science, design for disassembly to disassembly planning.

- When the expected life time of the product is high with respect to the supplier lead time, considering recovery operation as a primary source of satisfying demand does not make economical sense, even if the recovery operation is cost attractive and efficient.

Under these cases, considering items currently in use, and making purchasing decisions upon failures at recovery, increase costs in the long run. In addition when the recovery is not cost attractive and inefficient, the desired recovery probability is very low. In these cases, we expect that the exclusion of the items in process at the recovery operation will improve the performance of the systems. 
For a very restricted number of problems, inclusion of items in use in inventory position information improves costs. Since we assume that all of the items completing their life time return to the system, our model does not include any costs incurred for keeping track of these items. When the return process itself is stochastic in terms of the number of items return to the system, the source of recovery is smaller in terms of inflow to the recovery operation. Our result on the value of this piece of information in terms of costs and the sensitivity of the solutions with respect to demand rate (in our case it determines the inflow to the recovery operation in the long run) shows the importance of estimating the return intensity rather than potential returns in the future.

- We assume age independent success probability at recovery operation. In real life, we expect that as the age of the used items increase and the number of previous recoveries increase, the probability of success at recovery operation decreases. Similarly, the sensitivity of success with respect to increased recovery effort will be lower for the items used for a longer duration and already recovered before. In that sense, the desired recovery levels that we present constitute an upper bound for real life. Ferrer[2] already shows the importance of having the failure information as soon as possible. Under these cases, imposing rules restricting the age of the items that go through recovery operation will improve the system performance.

- We have ignored all possible effects of the workload on the throughput of recovery operation. When the resources carrying out recovery is limited in number, queueing effect occurs. In that case, effective lead times increase and consequently the inventory value of returns has more significant effect. We expect that when the recovery shop is capacitated, the desired level of recovery decreases compared to uncapacitated case.

\section{Appendix I -The Average Expected Cost Function under Policy I}

In this policy, it is proposed to make purchasing decision upon the completion of recovery operation. At each time an item fails in recovery, a new purchasing order of size 1 is given to the outside supplier. Therefore, this policy has an $(S-1, S)$ nature, and system-wide inventory position (as defined) is constant at level $S$. The routing of the parts can be modelled as a queuing network under Policy I. The steady state representation is given in Figure 3. In the 
figure, the nodes 0,1 and 2 represent the usage, recovery and purchasing operations. Since the demand process is Poisson and the usage time of each part is independent of the others', the node representing usage can be modelled as an $M / G / \infty$ queue. Due to the fact that, the departure process of an $M / G / \infty$ queue is Poisson with parameter equal to the parameter of the arrival process, the arrivals to recovery operation is also Poisson with parameter $\lambda$ in the steady-state. Therefore, the recovery operation can also be represented as an $M / G / \infty$ queue whose departures constitute a Poisson process with parameter $\lambda$ in the steady state. Since we assume the time stationarity of probability of success at recovery, the arrivals of orders to the supplier is Poisson with parameter $\left(1-p\left(T_{1}\right)\right) \lambda$ under inventory control policy employed. The additional assumption on the infiniteness of supplier's capacity enables us to model it as an $M / G / \infty$ queue, too.

\section{Place Figure 3 here}

In the steady state the number of items in an $M / G / \infty$ queue with an arrival process parameter $\lambda$ and mean service time parameter $1 / \mu$ is Poisson with parameter $\lambda / \mu$. (Kleinrock [3]). Therefore, expected holding cost of WIP can be expressed as,

$$
T C_{W I P}\left(S, T_{1}\right)=T C_{W I P}\left(T_{1}\right)=h_{1} \lambda T_{1}
$$

As it is also discussed in Section 2.1, in order to derive expected cost associated to serviceables, it is required to derive the steady state distribution of net inventory level of serviceables. Under Policy I, since every failure at recovery operation triggers an order arrival to the supplier, and there is no exit from the system, the sum of total number of items in the queuing network in Figure 3 and the serviceable net inventory is constant and equal to $S$. In other words, the total number in the nodes is equivalent to total number of outstanding orders. Therefore, if the total number in nodes 0,1 and $2, N=N_{0}+N_{1}+N_{2}$, is less than $S$, there are $S-N$ items in the serviceable inventory. On the other hand, if the total number, $N$ exceeds the base stock level $S$, this means that the backorder level is equal to $N-S$. Therefore, under Policy I, equation 2 can be re-written as,

$$
T C_{F G I}\left(S, T_{1}\right)=h\left(T_{1}\right) \sum_{i=0}^{S}(S-i) \cdot P(N=i)+b \sum_{i=S+1}^{\infty}(i-S) \cdot P(N=i) .
$$


Notice that, the random variables, $N_{0}, N_{1}$ and $N_{2}$ are independent. In the steady state the distribution of $N_{0}, N_{1}$ and $N_{2}$ are all Poisson with parameter $\lambda T_{0}, \lambda T_{1}$ and $\lambda\left(1-p\left(T_{1}\right)\right) T_{2}$. Therefore, the number of outstanding orders, $N$, is Poisson with parameter $\lambda\left[T_{0}+T_{1}+(1-\right.$ $\left.\left.p\left(T_{1}\right)\right) T_{2}\right]$, since it is the sum of three independent Poisson random variables.

Notice that the function $T C\left(S, T_{1}\right)$ reduces to a simple newsboy cost plus some constant terms for a given $T_{1} \geq 0$, and convex in $S \in\{1,2, \ldots\}$. Therefore, for a given value of $T_{1} \geq 0$, $S$ value minimizing $T C\left(S, T_{1}\right)$ can be found by,

$$
\min \left\{S: P\{N \leq S\} \geq \frac{b}{h\left(T_{1}\right)+b}\right\} .
$$

On the other hand, the convexity of $T C\left(S, T_{1}\right)$ with respect to $T_{1}>0$ for a given $S \in$ $\{1,2, \ldots\}$, and consequently joint convexity depends on the problem parameters.

Therefore we employ a full search procedure under which $p\left(T_{1}\right) \in[0,1)$ is searched with a finite step size. At each $p$ value considered the optimal $S$ value is found using 6 under the corresponding $T_{1}$ value. We select the best $\left(S, T_{1}\right)$ giving the minimum average cost.

\section{References}

1. de Brito MP. Managing Reverse Logistics or Reversing Logistics Management, PhD Dissertation, Erasmus Research Institute of Management, Erasmus University Rotterdam, 2004.

2. Ferrer G. Yield Information and Supplier Responsiveness in Remanufacturing Operations. European Journal of Operational Research 2003;149:540 556.

3. Kleinrock L. Queuing Systems, Vol. 1: Theory, John Wiley \& Sons, 1975.

4. Law AM, and Kelton WD. Simulation Modeling and Analysis, Mc Graw-Hill International Editions, 1991.

5. Rijneveld A. Optimizing Inventory Control Within SAP, MS Thesis, Econometric Institute, Erasmus University Rotterdam, 2001.

6. Teunter RH, van der Laan E, and Inderfurth K. How to set the holding cost rates in average cost inventory models with reverse logistics?. Omega, 2000; 28 (4):409-415. 
7. Teunter RH, and van der Laan E. On the non-optimality of the average cost approach for inventory models with remanufacturing. International Journal of Production Economics, $2002 ; 79(1), 67-73$.

8. van der Laan E. An NPV and AC analysis of a stochastic inventory system with joint manufacturing and remanufacturing, International Journal of Production Economics, 2003; 81-82(11) 317-331.

9. www.opttek.com

Table 1: Notation used

\begin{tabular}{|rl|}
\hline$\lambda:$ & demand rate \\
\hline$T_{0}:$ & expected usage time of an item \\
\hline$T_{1}:$ & expected time spent for recovery operation \\
\hline$T_{2}:$ & expected supplier lead time \\
\hline$h_{1}:$ & unit holding cost of WIP at recovery operation \\
\hline$h\left(T_{1}\right):$ & unit holding cost of serviceables for given value of $T_{1}$ \\
\hline$b:$ & unit backorder cost \\
\hline$c_{r}\left(T_{1}\right):$ & unit recovery cost for given value of $T_{1}$ \\
\hline$c_{p}:$ & unit cost of purchasing \\
\hline$r:$ & inventory carrying charge \\
\hline$N_{0}:$ & number of items currently in use \\
\hline$N_{1}:$ & number of items under recovery operation \\
\hline$N_{2}:$ & number of items on-order from the supplier \\
\hline$B:$ & backorder level in the serviceable inventory \\
\hline$O H:$ & on-hand stock in the serviceable inventory \\
\hline
\end{tabular}


Table 2: $k_{p}$ and $k_{c}$ Values for the Cases Depicted in Figures 1 and 2

\begin{tabular}{|c|c|c|c|c|}
\hline Case: & 1 & 2 & 3 & 4 \\
\hline$k_{p}$ & 0.5 & 0.5 & 2 & 2 \\
\hline$k_{c}$ & 0.5 & 2 & 0.5 & 2 \\
\hline
\end{tabular}

Table 3: Summary of Inventory Control Policies

\begin{tabular}{|c|c|c|}
\hline & $I P_{1}$ & $I P_{2}$ \\
\hline$E_{1}$ & Policy I & Policy II \\
\hline$E_{2}$ & Policy III & Policy IV \\
\hline
\end{tabular}

Table 4: Levels of the Factors

\begin{tabular}{|c|c|c|c|c|c|c|c|}
\hline Factor: & $k_{p}$ & $k_{c}$ & $T_{0}$ & $T_{2}$ & $c_{b}$ & $\lambda$ & $h_{1}$ \\
\hline Levels: & $0.5,2$ & $0.5,2$ & 5,100 & 0,3 & $0.5,0.1$ & $0.1,0.01$ & $0,0.1$ \\
\hline
\end{tabular}



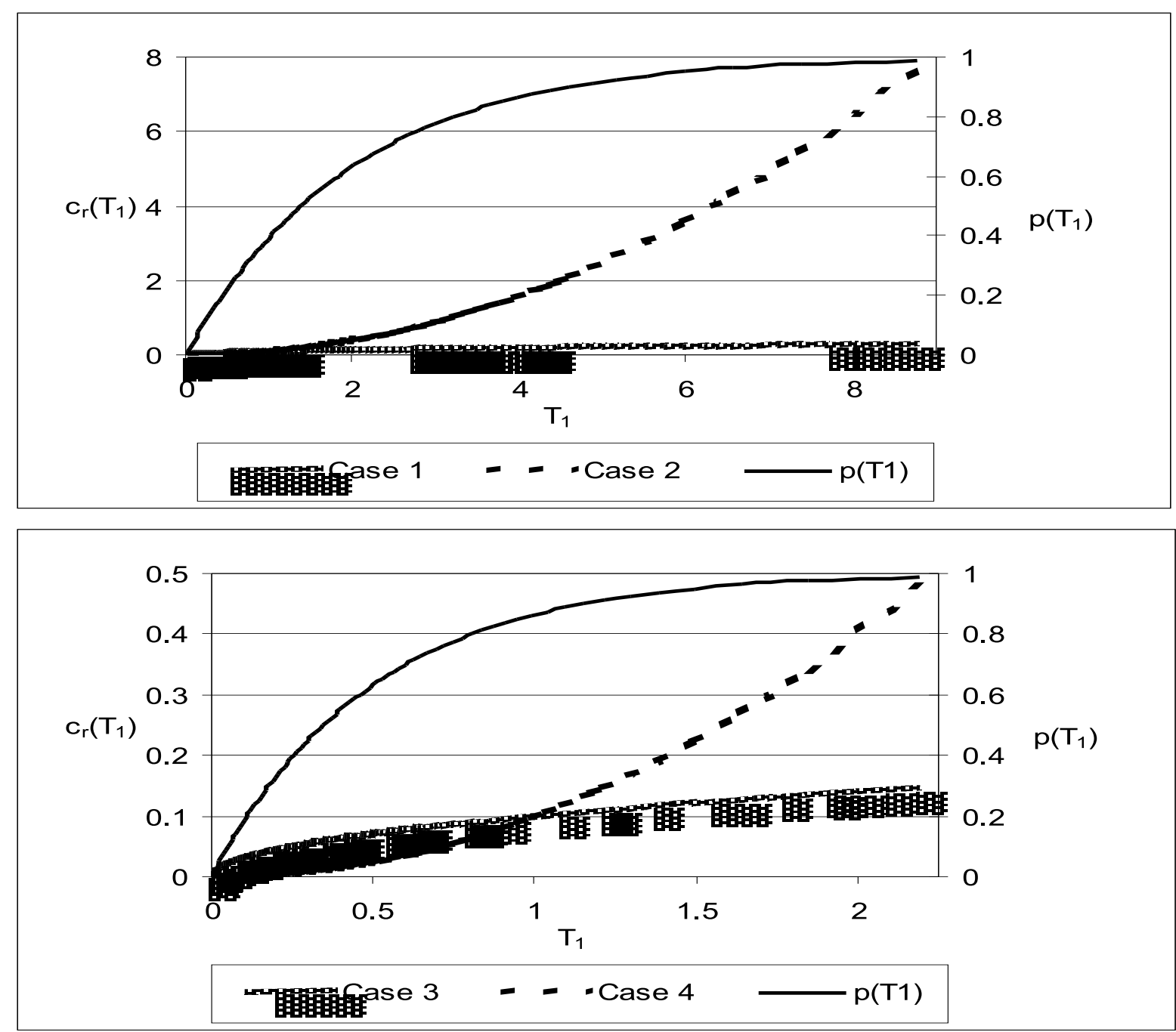

Figure 1: Unit Recovery Cost and Recovery Probability under Different Recovery Effort Levels and Cost/Efficiency Conditions $c_{b}=0.1$ 

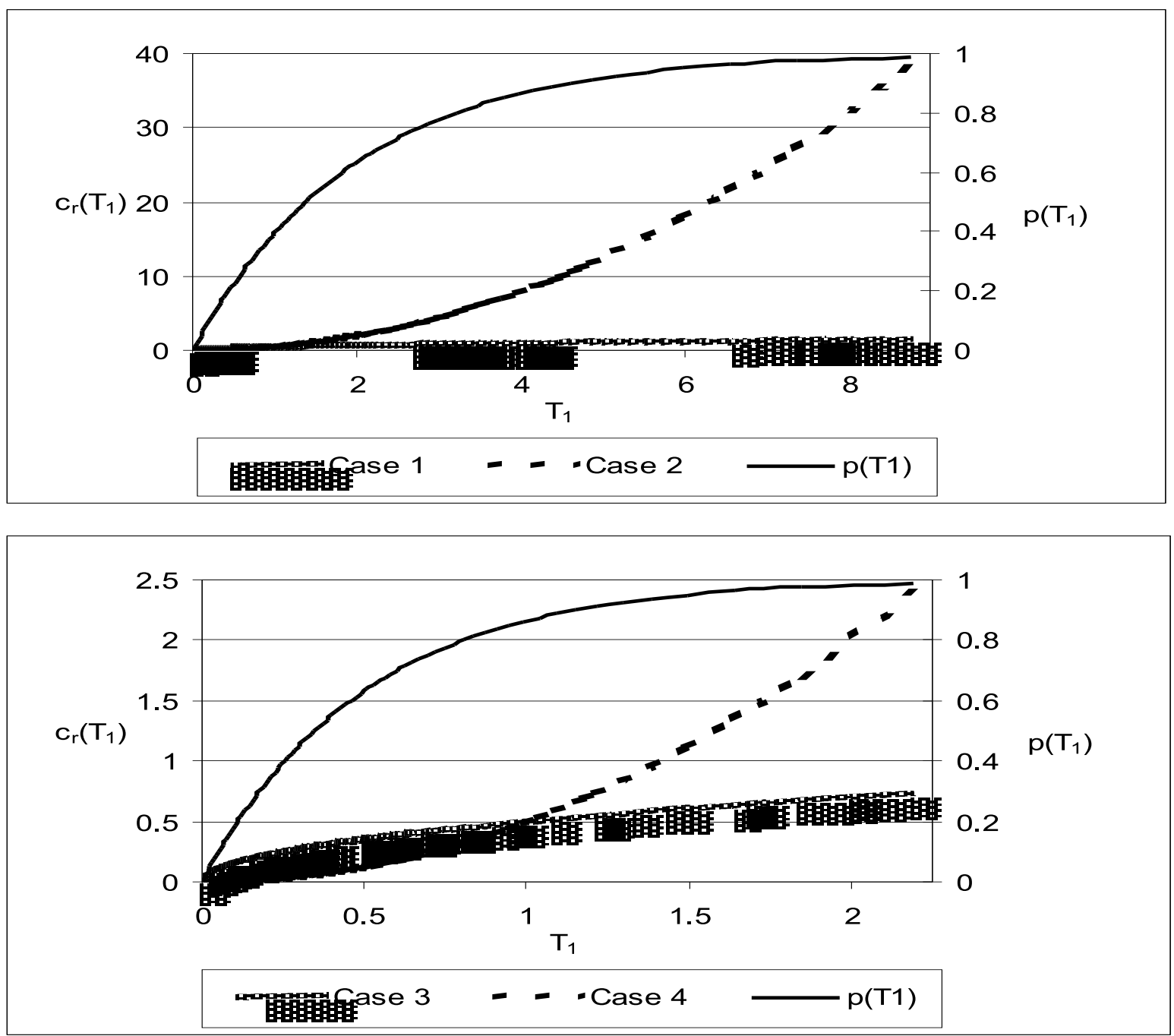

Figure 2: Unit Recovery Cost and Recovery Probability under Different Recovery Effort Levels and Cost/Efficiency Conditions $c_{b}=0.5$ 


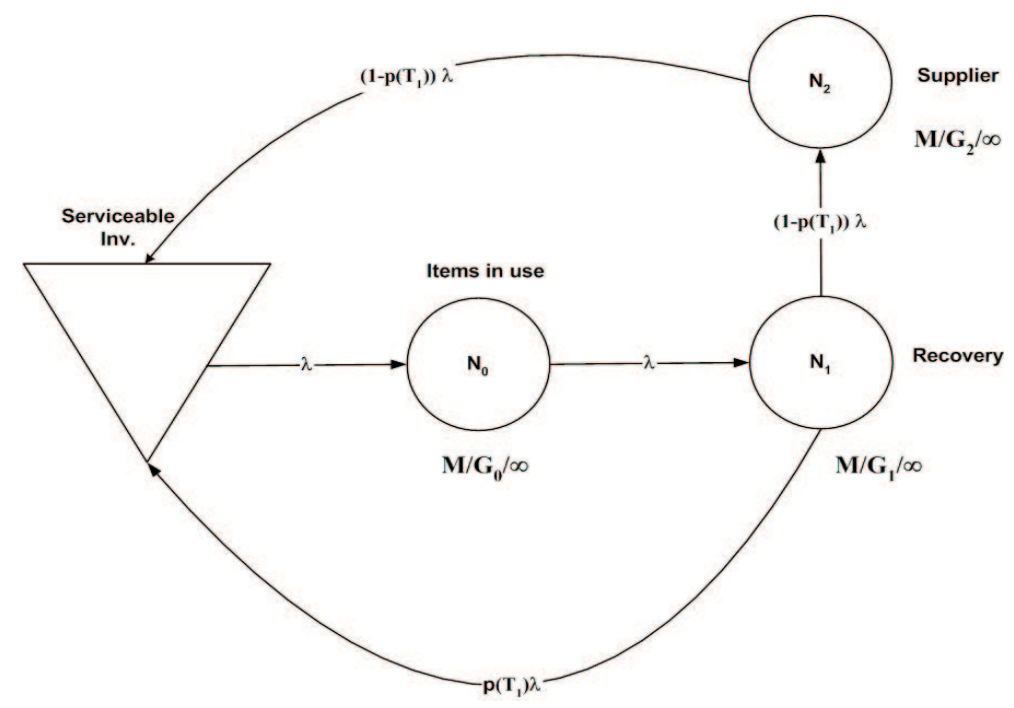

Figure 3: The Picture of the System in the Steady State

Table 5: Percentage Cost Improvement by the Best Policy over the Worst One $\left(T_{0}=100\right)$

\begin{tabular}{|r|r||r|r||c|c||c|c||c|c|}
\hline Case & & $T_{2}=0$ & $T_{2}=3$ & $c_{b}=0.1$ & $c_{b}=0.5$ & $h_{1}=0$ & $h_{1}=0.1$ & $\lambda=0.01$ & $\lambda=0.1$ \\
\hline \hline 1 & $\min$ & 33.41 & 32.54 & 32.54 & 63.95 & 32.54 & 63.26 & 32.54 & 48.97 \\
\hline & $\max$ & 84.56 & 73.74 & 80.60 & 84.56 & 80.81 & 84.56 & 69.20 & 84.56 \\
\hline & average & 67.20 & 61.09 & 56.45 & 71.84 & 56.84 & 71.45 & 57.49 & 70.79 \\
\hline \hline 2 & $\min$ & 57.23 & 56.81 & 56.81 & 63.38 & 56.81 & 65.22 & 56.81 & 67.44 \\
\hline & $\max$ & 83.78 & 72.85 & 82.55 & 83.78 & 82.37 & 83.78 & 67.82 & 83.78 \\
\hline \hline & average & 72.69 & 66.89 & 68.03 & 71.55 & 67.59 & 71.99 & 63.36 & 76.22 \\
\hline 3 & $\min$ & 28.77 & 23.87 & 23.87 & 53.97 & 23.87 & 60.27 & 23.87 & 37.17 \\
\hline & $\max$ & 83.32 & 71.45 & 77.31 & 83.32 & 75.02 & 83.32 & 67.07 & 83.32 \\
\hline \hline & average & 61.87 & 54.82 & 49.86 & 66.83 & 47.93 & 68.76 & 52.08 & 64.62 \\
\hline & $\min$ & 38.85 & 38.06 & 38.06 & 49.22 & 38.06 & 60.12 & 38.06 & 51.91 \\
\hline & $\max$ & 80.81 & 68.33 & 77.60 & 60.12 & 73.89 & 62.71 & 64.19 & 80.81 \\
\hline & average & 63.58 & 57.31 & 56.69 & 54.16 & 53.42 & 61.50 & 53.19 & 67.71 \\
\hline
\end{tabular}


Table 6: Percentage Cost Improvement by the Best Policy over the Worst One $\left(T_{0}=5\right)$

\begin{tabular}{|r|r||r|c||c|c||c|c||c|c|}
\hline Case & & $T_{2}=0$ & $T_{2}=3$ & $c_{b}=0.1$ & $c_{b}=0.5$ & $h_{1}=0$ & $h_{1}=0.1$ & $\lambda=0.01$ & $\lambda=0.1$ \\
\hline \hline 1 & $\min$ & 4.66 & 0.74 & 0.74 & 3.53 & 1.17 & 0.74 & 0.74 & 3.21 \\
\hline & $\max$ & 53.17 & 31.40 & 46.03 & 53.17 & 53.17 & 53.17 & 15.15 & 53.17 \\
\hline & average & 24.24 & 12.73 & 13.88 & 23.09 & 25.31 & 21.67 & 6.55 & 30.42 \\
\hline \hline 2 & $\min$ & 2.02 & 1.69 & 3.06 & 1.69 & 1.69 & 2.02 & 1.69 & 20.08 \\
\hline & $\max$ & 50.28 & 27.64 & 46.84 & 50.28 & 44.71 & 50.28 & 13.10 & 50.28 \\
\hline \hline & average & 26.48 & 15.24 & 20.16 & 21.57 & 20.24 & 21.48 & 6.13 & 35.59 \\
\hline 3 & $\min$ & 1.17 & 0.74 & 0.99 & 1.17 & 0.99 & 1.17 & 1.69 & 0.99 \\
\hline & $\max$ & 48.00 & 31.40 & 29.54 & 48.00 & 30.78 & 48.00 & 13.10 & 48.00 \\
\hline \hline & average & 24.12 & 12.73 & 17.07 & 19.76 & 18.05 & 18.78 & 6.13 & 19.32 \\
\hline & $\min$ & 13.43 & 2.20 & 2.20 & 9.71 & 2.20 & 7.65 & 9.28 & 2.20 \\
\hline & $\max$ & 38.27 & 21.76 & 28.97 & 38.27 & 33.70 & 38.27 & 23.45 & 38.27 \\
\hline & average & 25.18 & 11.70 & 16.65 & 20.23 & 17.51 & 19.37 & 16.57 & 20.32 \\
\hline
\end{tabular}


Table 7: Desired Recovery Probability $\left(T_{0}=100\right)$

\begin{tabular}{|r|r||r|c||c|c||c|c||c|c|}
\hline Case & & $T_{2}=0$ & $T_{2}=3$ & $c_{b}=0.1$ & $c_{b}=0.5$ & $h_{1}=0$ & $h_{1}=0.1$ & $\lambda=0.01$ & $\lambda=0.1$ \\
\hline \hline 1 & $\min$ & 0.00 & 0.00 & 0.00 & 0.00 & 0.00 & 0.00 & 0.00 & 0.00 \\
\hline & $\max$ & 0.84 & 0.82 & 0.84 & 0.22 & 0.84 & 0.21 & 0.84 & 0.80 \\
\hline & average & 0.21 & 0.26 & 0.41 & 0.05 & 0.44 & 0.03 & 0.21 & 0.26 \\
\hline \hline 2 & $\min$ & 0.00 & 0.00 & 0.00 & 0.00 & 0.01 & 0.00 & 0.00 & 0.01 \\
\hline & $\max$ & 0.44 & 0.47 & 0.47 & 0.23 & 0.47 & 0.20 & 0.44 & 0.47 \\
\hline \hline & average & 0.13 & 0.17 & 0.23 & 0.06 & 0.26 & 0.04 & 0.11 & 0.19 \\
\hline & $\min$ & 0.00 & 0.00 & 0.00 & 0.00 & 0.00 & 0.00 & 0.00 & 0.00 \\
\hline & max & 0.95 & 0.95 & 0.95 & 0.46 & 0.95 & 0.39 & 0.95 & 0.92 \\
\hline \hline 4 & average & 0.27 & 0.34 & 0.51 & 0.10 & 0.56 & 0.05 & 0.24 & 0.37 \\
\hline & $\max$ & 0.00 & 0.00 & 0.00 & 0.00 & 0.53 & 0.00 & 0.00 & 0.02 \\
\hline & average & 0.34 & 0.44 & 0.46 & 0.32 & 0.69 & 0.09 & 0.35 & 0.44 \\
\hline
\end{tabular}


Table 8: Desired Recovery Probability $\left(T_{0}=5\right)$

\begin{tabular}{|r|r||r|c||c|c||c|c||c|c|}
\hline Case & & $T_{2}=0$ & $T_{2}=3$ & $c_{b}=0.1$ & $c_{b}=0.5$ & $h_{1}=0$ & $h_{1}=0.1$ & $\lambda=0.01$ & $\lambda=0.1$ \\
\hline \hline 1 & $\min$ & 0.00 & 0.00 & 0.00 & 0.00 & 0.00 & 0.00 & 0.00 & 0.00 \\
\hline & $\max$ & 0.93 & 0.95 & 0.95 & 0.36 & 0.95 & 0.66 & 0.95 & 0.93 \\
\hline & average & 0.24 & 0.42 & 0.60 & 0.06 & 0.51 & 0.15 & 0.32 & 0.33 \\
\hline \hline 2 & $\min$ & 0.00 & 0.01 & 0.00 & 0.01 & 0.18 & 0.00 & 0.01 & 0.00 \\
\hline & $\max$ & 0.43 & 0.53 & 0.53 & 0.30 & 0.53 & 0.34 & 0.53 & 0.51 \\
\hline \hline & average & 0.14 & 0.26 & 0.25 & 0.15 & 0.33 & 0.07 & 0.15 & 0.25 \\
\hline & $\min$ & 0.00 & 0.00 & 0.00 & 0.00 & 0.01 & 0.00 & 0.00 & 0.00 \\
\hline & $\max$ & 0.97 & 0.98 & 0.98 & 0.85 & 0.98 & 0.91 & 0.98 & 0.98 \\
\hline \hline 4 & average & 0.31 & 0.65 & 0.71 & 0.26 & 0.73 & 0.23 & 0.43 & 0.54 \\
\hline & $\min$ & 0.00 & 0.65 & 0.01 & 0.00 & 0.64 & 0.00 & 0.01 & 0.00 \\
\hline & average & 0.45 & 0.76 & 0.71 & 0.50 & 0.77 & 0.43 & 0.57 & 0.63 \\
\hline
\end{tabular}


Table 9: Optimal $T_{1}$ values $\left(T_{0}=100\right)$

\begin{tabular}{|r|r||r|c||c|c||c|c||c|c|}
\hline Case & & $T_{2}=0$ & $T_{2}=3$ & $c_{b}=0.1$ & $c_{b}=0.5$ & $h_{1}=0$ & $h_{1}=0.1$ & $\lambda=0.01$ & $\lambda=0.1$ \\
\hline \hline 1 & $\min$ & 0.00 & 0.00 & 0.00 & 0.00 & 0.00 & 0.00 & 0.00 & 0.00 \\
\hline & $\max$ & 3.60 & 3.43 & 3.60 & 0.49 & 3.60 & 0.47 & 3.60 & 3.20 \\
\hline & average & 0.75 & 0.95 & 1.58 & 0.12 & 1.64 & 0.06 & 0.88 & 0.82 \\
\hline \hline 2 & $\min$ & 0.01 & 0.01 & 0.00 & 0.01 & 0.02 & 0.01 & 0.01 & 0.03 \\
\hline & $\max$ & 1.15 & 1.27 & 1.27 & 0.52 & 1.27 & 0.46 & 1.15 & 1.27 \\
\hline \hline & average & 0.31 & 0.43 & 0.69 & 0.14 & 0.65 & 0.09 & 0.27 & 0.46 \\
\hline & $\min$ & 0.00 & 0.00 & 0.01 & 0.00 & 0.00 & 0.00 & 0.00 & 0.00 \\
\hline & $\max$ & 1.46 & 1.46 & 1.27 & 0.31 & 1.46 & 0.25 & 1.46 & 1.23 \\
\hline \hline & average & 0.33 & 0.41 & 0.60 & 0.06 & 0.71 & 0.03 & 0.37 & 0.37 \\
\hline & $\min$ & 0.00 & 0.00 & 0.00 & 0.00 & 0.38 & 0.00 & 0.00 & 0.01 \\
\hline & average & 0.93 & 0.88 & 0.93 & 0.46 & 0.93 & 0.25 & 0.93 & 0.88 \\
\hline
\end{tabular}


Table 10: Optimal $T_{1}$ values $\left(T_{0}=5\right)$

\begin{tabular}{|r|r||r|c||c|c||c|c||c|c|}
\hline Case & & $T_{2}=0$ & $T_{2}=3$ & $c_{b}=0.1$ & $c_{b}=0.5$ & $h_{1}=0$ & $h_{1}=0.1$ & $\lambda=0.01$ & $\lambda=0.1$ \\
\hline \hline 1 & $\min$ & 0.00 & 0.00 & 0.00 & 0.00 & 0.00 & 0.00 & 0.00 & 0.00 \\
\hline & $\max$ & 5.44 & 5.80 & 5.80 & 0.90 & 5.80 & 2.18 & 5.80 & 5.44 \\
\hline & average & 1.21 & 1.83 & 2.90 & 0.14 & 2.59 & 0.44 & 1.53 & 1.51 \\
\hline \hline 2 & $\min$ & 0.00 & 0.02 & 0.00 & 0.01 & 0.38 & 0.00 & 0.01 & 0.00 \\
\hline & $\max$ & 1.11 & 1.50 & 1.50 & 0.74 & 1.50 & 0.82 & 1.50 & 1.43 \\
\hline \hline & average & 0.34 & 0.68 & 0.66 & 0.36 & 0.86 & 0.16 & 0.39 & 0.63 \\
\hline & $\min$ & 0.00 & 0.00 & 0.00 & 0.00 & 0.00 & 0.00 & 0.00 & 0.00 \\
\hline & $\max$ & 1.81 & 1.93 & 1.93 & 0.93 & 1.93 & 1.22 & 1.93 & 1.84 \\
\hline \hline 4 & average & 0.48 & 0.97 & 1.18 & 0.28 & 1.18 & 0.28 & 0.71 & 0.75 \\
\hline & $\min$ & 0.00 & 0.52 & 0.00 & 0.00 & 0.51 & 0.00 & 0.00 & 0.00 \\
\hline & average & 0.44 & 0.80 & 0.81 & 0.42 & 0.81 & 0.42 & 0.59 & 0.64 \\
\hline
\end{tabular}

